\title{
ACCEPTANCE AS \\ CONDITIONAL DISPOSITION
}

FABIO PAGLIERI

Istituto di Scienze e Tecnologie della Cognizione - CNR, Roma

\begin{abstract}
The notion of acceptance has a checkered history in philosophy. This paper discusses what version of acceptance, if any, should qualify for inclusion in epistemology. The inquiry is motivated by van Fraassen's invitation to be more liberal in determining basic epistemological categories (section 1). Reasons are given to avoid extending this liberal attitude to include van Fraassen's acceptance of scientific theories (section 2) and Bratman's pragmatic acceptance (section 3): both notions are showed to be reducible to combinations of simpler constitutive elements, and thus useful only as a shorthand. Other cases of divergence between action and belief, due to automatic sub-personal routines, are also not liable of being interpreted as acceptances (section 4). Only acceptance of conditional statements is argued to have something solid to offer for epistemological purposes: in particular, discussion on accepting conditional statements serves as a springboard to develop a new understanding of acceptance in general (section 5). It is proposed to consider acceptance as a conditional disposition: the consequences of this view for epistemology are discussed (section 6).
\end{abstract}

\section{INTRODUCTION}

Bas van Fraassen recently urged us to be more liberal in our epistemological explorations: "Epistemology has in the past been guilty of a really big sin of omission. There is in fact an enormous variety of epistemic attitudes, with many nuances and distinctions to be drawn. Prior to epistemological controversies we should have had a descriptive epistemology, to canvass this variety. What we have instead at this point is a patchwork, to which items are proposed for addition or deletion from time to time. [...] But traditionalists were and are guilty of the opposite extremism. They write as if belief, disbelief, and neutrality are the only epistemic attitudes there are" (2001, p.165). There are two ways to go, if we are to endorse van Fraassen's invitation: atomism and molecularism. An atomist epistemology is one in which new primitive epistemic attitudes are considered for inclu- 
sion, on a par with more traditional notions such as belief and knowledge. In a molecular epistemology, new epistemic attitudes are conceived as molar notions, defined as stable combinations of more primitive elements that present specific functional properties, possibly not reducible to those of their parts. As far as epistemology is concerned, these alternatives are not mutually exclusive: epistemic categories can be expanded both by defining new primitives, and by describing stable and relevant combinations of them. However, when considering a single attitude as a candidate, a decision need to be made: Is it a primitive notion or a molar one?

A prime candidate for van Fraassen's descriptive epistemology is the notion of acceptance, that over the past decades cropped up in many philosophical fields, each with its own variety: in philosophy of science, defining acceptance of a scientific theory is a basic concern of constructive empiricism (van Fraassen 1980; 2001); in philosophy of action, pragmatic acceptance is proposed as a cognitive attitude guiding practical reasoning and action (Bratman 1992); in philosophy of mind, acceptance is a key concept in the debate on collective mental states (Tuomela 2000; Wray 2001; Gilbert 2002); and in philosophy of language and logic, acceptance of conditional statements is the crux of the Ramsey Test and its subsequent elaborations (Ramsey 1929; Stalnaker 1968; Lewis 1976; 1986; Gärdenfors 1986; Leitgeb 2007). More in general, insofar as acceptance is broadly conceived as a cognitive attitude, determining its exact status is a chief concern for philosophy of mind, with ancillary benefits for other fields where the notion plays a role.

This paper addresses two questions on the status of acceptance: "Is acceptance either a primitive or a molar notion?", and "Is acceptance a truly epistemic attitude?". The answers to these questions are intertwined: it is argued that acceptance can be conceived either as a molar attitude with both epistemic and conative components (in which case it is not particularly heuristic), or, more interestingly, as a primitive transformational attitude. Depending on what definition one endorses, acceptance is either reducible to a finer epistemological grain or not. This clearly requires qualification, in light of the various meanings of "acceptance". Indeed, part of the challenge is to identify significant connections among some of these meanings, in spite of their apparent heterogeneity. 


\section{ACCEPTANCE IN CONSTRUCTIVE EMPIRICISM}

In The Scientific Image, van Fraassen suggests that accepting a scientific theory as valid does not coincide with believing it to be literally true, but rather empirically adequate: "A theory is empirically adequate if what it says about the observable things and events in this world is true - exactly if it 'saves the phenomena'. A little more precisely: such a theory has at least one model that all the actual phenomena fit inside. I must emphasize that this refers to all the phenomena; these are not exhausted by those actually observed" (1980, p.12). The implication is that whatever the theory says about events or processes that are not (and never will be) observable need not be believed by those who endorse the theory. But this is not the whole story: "Acceptance of theories [...] is a phenomenon of scientific activity which clearly involves more than belief. One main reason for this is that we are never confronted with a complete theory. So if a scientist accepts a theory, he thereby involves himself in a certain sort of research programme. [...] Thus acceptance involves not only belief but a certain commitment $[\ldots]$ to confront any future phenomena by means of the conceptual resources of this theory. [...] A commitment is of course not true or false: The confidence exhibited is that it will be vindicated" (ibid., pp.1213). This commitment is what van Fraassen calls the pragmatic dimension of acceptance.

Let it be noted in passing that doing justice to van Fraassen's constructive empiricism is not the purpose here: it is enough to assess whether his notion of acceptance is primitive or molar, and whether it is purely epistemic or not. In this respect, the verdict is easy to reach: theory acceptance combines a general belief in empirical adequacy (which entails several specific beliefs on concrete instantiations of that adequacy), plus a pragmatic commitment to perform certain actions, given the appropriate conditions (e.g., confronting new data with the theory's predictions, striving to further detail the theory itself, being open to controversy on it, etc.). This defines acceptance as a molar notion, which combines both epistemic and conative ingredients.

A more subtle issue is whether this concept deserves hospitality in descriptive epistemology, and why. It certainly serves as a useful shorthand, because the notion (assuming van Fraassen is right) elegantly captures our attitude towards a large class of phenomena, i.e. scientific theories, and being reducible to simpler ingredients does not diminish this practical value. But is this enough to justify relaxing the traditional austerity of 
our epistemological categories? After all, another way of putting van Fraassen's proposal is to say that (i) belief in the empirical adequacy of a theory generates a certain commitment to vindicate it, but (ii) such a belief does not imply belief in the truth of the theory or in the reality of the entities it postulates, whereas the converse is true. This characterization, plus the psychological hypothesis that belief in empirical adequacy is what matters in assessing scientific theories, approximates well the basic tenets of constructive empiricism, with no need to mention acceptance. We can still use it for the sake of brevity, of course, but this seems pretty much the whole extent of its heuristic value.

Moreover, there are good reasons to consider van Fraassen's notion of acceptance as specifically tailored to scientific theories, and to reserve its use for that purpose - which further limits its general significance. Imagine in contrast to extend the scope of application of van Fraassen's acceptance beyond scientific theories to factual statements. The suggestion would be to conceive factual statements like "Barack Obama is a smart man", or "The brain is the seat of the soul", as theories-in-a-nutshell, so that accepting them (as opposed to believing them) would consist of believing in their empirical adequacy and having a commitment to vindicate them: e.g, the subject would believe that Obama will not be outsmarted by political adversaries, or that dualists are mistaken on the nature of consciousness, and will be ready to confront any future phenomena by means of the conceptual resources derived from the accepted statements.

The problem with extending the notion this way, and the reason why van Fraassen would probably not subscribe to it, is that in general it is very hard to justify the claim that accepting a factual statement does not also implies believing it to be true, rather than just empirically adequate. Is it possible to accept that "Barack Obama is a smart man" without believing it to be true? With the exceptions of few limiting cases (discussed in the next section), the answer seems to be negative. This is because factual statements usually do not involve appeal to unobservable postulates and are not inherently incomplete, contrary to what happens with scientific theories. Since factual statements can typically uphold the stronger standards of belief, there is no reason to invoke the weaker attitude of acceptance.

To sum up: acceptance in constructive empiricism is a molar notion of spurious composition, including both epistemic and conative elements, i.e. beliefs plus commitments. While quite handy for easiness of reference, it does not add anything specific to the alchemy of its primitive components, and its significance is best confined to discussion of scientific theo- 
ries: thus its limited appeal for inclusion in a general descriptive epistemology.

\section{DECONSTRUCTING BRATMAN:}

WHY PRAGMATIC ACCEPTANCE IS NOT AN EPISTEMIC PRIMITIVE

Stalnaker (1984) introduced the notion of acceptance as a technical term, in the context of his analysis of inquiry, to identify a broad class of propositional attitudes of which belief is just a member.

Acceptance, as I shall use this term, is a broader concept than belief; it is a generic propositional attitude concept with such notions as presupposing, presuming, postulating, positing, assuming and supposing falling under it. [...] To accept a proposition is to treat it as a true proposition in one way or another - to ignore, for the moment at least, the possibility that it is false. [...] To accept a proposition is to act, in certain respects, as if one believed it (Stalnaker 1984, pp.79-80, my emphasis).

Two points deserve special consideration: first, this notion is linked, by definition, with the practical usage of a representation (the accepted proposition is treated as true; the individual acts in certain respects as if it were indeed held to be true), and thus it is usually labelled "pragmatic acceptance"; second, the notion is deemed to be broader than belief, so that the class of acceptances encompasses the class of beliefs as one of its subsets. While the former claim is largely uncontroversial, the latter is a matter of debate. According to Stalnaker, to believe something would imply accepting it as well, because the first notion is just a specification of the second. This view, however, is at odds with cases where the agent's beliefs are not acted upon, i.e. are not pragmatically accepted. In certain situations it may be prudent to behave in accordance with a conception of the world that deviates from the agent's beliefs in two ways: accepting something without believing it, and refusing to accept something that is believed. Consider the following example, due to Bratman:

I plan for a major construction project to begin next month. I need to decide now whether to do the entire project at once or instead to break the project into two parts, to be executed separately. The rationale for the second strategy is that I am unsure whether I presently have the financial resources to do the whole thing at once. I know that in the case of each sub-contractor - carpenter, plumber, and so on - it is only possible at present to get an estimate of the range 
of potential costs. In the face of this uncertainty I proceed in a cautious way: In the case of each sub-contractor I take it for granted that the total costs will be at the top of the estimated range. On the basis of these assumptions I determine whether I have at present enough money to do the whole project at once. In contrast, if you offered me a bet on the actual total cost of the project - the winner being the person whose guess is closer to the actual total - I would reason differently (Bratman 1992, p.6).

Similarly, in certain neighbourhoods of large metropolitan areas it may be prudent to act as if any passer-by is a potential bag snatcher, even if one does not really believe that this is likely - i.e., you would not be ready to denounce any of them to the police without further evidence. In such situations, subjects seem to accept something which they do not believe, and also (against Stalnaker's claim) to believe something that they do not accept, i.e. that they are unwilling to use as a basis for action. In the construction example, the subject forms an estimate of the most likely total cost of the construction work, but he does not act on the basis of this estimate. In order to account for cases of "believing without accepting" and "accepting without believing", some authors (Cohen 1989; Bratman 1992; Tuomela 2000) argue that, contrary to the view of Stalnaker and others (Engel 1998; Wray 2001), beliefs and acceptances are closely related but mutually independent concepts, neither of which entails the other. The rest of this section is devoted to discuss such view, while Stalnaker's position will be considered in section 5, on the acceptance of conditionals.

An independentist view of pragmatic acceptance invites to consider this notion as an epistemic primitive, on a par with belief but distinct from it. Bratman (1992) championed this proposal, indicating in the sensitivity to context the main difference between belief and acceptance, and the reason for including the latter in his analysis of practical reasoning. Whereas what we rationally accept can change across contexts, belief is supposed to be context-independent: it is not typically considered reasonable to have belief $p$ relative to context $X$ but not with respect to context $Y$ (Bratman 1992, p.3). To further show that acceptance is not reducible to belief, Bratman discusses a garden variety of cases where actions and beliefs part ways, and argue that similar cases require us to make use of the notion of acceptance to analyze the agent's practical reasoning. All the examples are gathered under five categories: (1) simplification of one's reasoning; (2) asymmetries in the costs of errors; (3) needs of social cooperation; (4) special relations to others; (5) pre-conditions for any practical reasoning at all. For reasons of space, here only examples in categories (2), (4), and (5) will be 
discussed, with the aim of showing that all these situations can easily be explained without making use of the notion of acceptance, thus they fail to justify treating acceptance as a genuine epistemic primitive, contra Bratman. Similar considerations apply also to cases under (3), whereas instances of (1) do not provide any conclusive reason for the distinction between belief and acceptance to start with, as Bratman admits (1992, p.6).

The construction example mentioned above is a typical case of acceptance motivated by asymmetries in the costs of error. But is it possible to explain similar cases (i) without invoking any independent notion of acceptance, and yet (ii) saving the intuition that here the subject is acting sensibly, regardless any apparent divergence between beliefs and actions? Indeed, the construction example is clearly amenable of an alternative reconstruction: we could argue that here the subject is simply acting on a complex set of beliefs, in view of several intertwined goals. More precisely, he believes it to be unlikely that the costs will be at the top of the range, and yet he also believes it to be possible (although remotely) for them to skyrocket to that height - in fact, if he did not believe possible for such a thing to occur, he would have no reason to act as he does, and our intuition on his rationality would waver. ${ }^{1}$ Moreover, he believes that, if the costs should levitate too much, then he would be in deep financial troubles, and he wants to avoid that, even if he intends to realize the construction project within a reasonable amount of time. Given these motives and these beliefs, he acts as he does, and we consider him to be rationally justified in doing so. This seems a reasonable explanation of this case, one in which acceptances do not feature at all.

The same reasoning applies to any kind of behaviour dictated by prudential reasons. In the bag-snatcher example mentioned before, my overly suspicious behaviour towards passer-bys does not need to indicate that I am accepting that each of them is a potential bag-snatcher, although I do not really believe it. Instead, I am simply acting on the belief that it is at least possible to be robbed in certain metropolitan areas, and since I have a strong desire to avoid that happening to me, I stick to a 'better-safe-than-

\footnotetext{
${ }^{1}$ Alternatively, the case could be construed as involving a general belief to the effect that "Shit happens!", so that being prudent whenever something very important is at stake is rational as a general policy, rather than as a specific strategy for this particular problem. Even so, there is no need to invoke any notion of acceptance: the situation can be explained in terms of the interplay between specific and general beliefs, modulated by the agent's concerns, i.e. how much he cares about, respectively, quickly finishing the construction project and avoiding the risk of bankruptcy.
} 
sorry' policy. Given that prudential strategies are usually coupled with some uncertainty on future outcomes, they can be easily explained by bringing into the picture beliefs on possibilities and the desire of avoiding the most bleak of those possibilities, rather than acceptances.

Similar considerations apply also to different cases. Take two other examples from Bratman, concerning, respectively, special personal relation with someone, and precondition to any further practical reasoning:

My close friend has been accused of a terrible crime, the evidence of his guilt is strong, but my friend insists on his innocence. Despite the evidence of guilt, my close friendship may argue for assuming, in my ordinary practical reasoning and action, that he is innocent of the charge. In making plans for a dinner party, for example, such considerations of loyalty might make it reasonable for me to take his innocence for granted and so not use this issue to preclude inviting him. Yet if I find myself on the jury I may well think that I should not take his innocence for granted in that context for reasons of friendship.

A soldier in a war zone has his doubts that he will make it through the day and expresses these doubts in a letter he writes in the morning. Nevertheless, after writing his letter he proceeds to make plans for his daily tour of the battlefield; and in so doing it takes it for granted that he will be around to execute these plans. After all, how else could he plan for the day? Since he needs to make such an assumption in order to get his planning off the ground, such acceptance may be reasonable even in the face of his doubts (Bratman 1992, p.8).

Both examples can be convincingly explained without mentioning acceptance. In the first case, different standards clearly depends on different motives: while planning the dinner party, my main concern is towards my friend, and I want to be fair and loyal to him, not being completely sure of his guilt; whereas, once I am called upon to pass judgement on his conduct in a court of law, I feel compelled to assess the matter as objectively as I can, since my goal is to reach a true conclusion on the charge against him. My beliefs concerning the possibility of his guilt can be the same in both contexts; it is enough that I am driven by different motives, to account for the apparent incongruence in my behaviour. As for the second case, here the soldier's decision to carry on with his routine for the day, despite bleak prospects for survival, appears rational to us precisely because of the system of beliefs and goals that supports this choice. He seems convinced that giving in to despair would prevent him from performing his duty, and he may well be determined to avoid this disgrace. In fact, if he had a different set of mind, he should act differently: if he was insensitive to the call of duty and inclined to depressive brooding, it would be reasonable for him 
not to make anything and simply lay low in some trench, waiting for the worst to happen. The proper way of understanding his behaviour is not to contrive a stipulative notion of acceptance, but to look more carefully at the complex set of beliefs and goals that makes his conduct reasonable.

In the end, many alleged examples of the independence of acceptance from belief have an alternative explanation, one that is equally heuristics, more general, and more economical - insofar as it avoids introducing an additional epistemic item. Following van Fraassen's advice, we may wish to be more liberal in epistemology, but certainly we do not want to be careless, introducing a new primitive when there is no apparent need for it. This said, it remains possible to keep the label "pragmatic acceptance" as a shorthand for indicating a structure of beliefs and goals such that the agent seems to act in violation of some other beliefs. But this label would be of dubious value. For start, we lack a precise definition of what structures of beliefs and goals would qualify: it is one thing to show that Bratman's examples are liable of intuitive explanations in terms of beliefs and goals, it is another matter to extrapolate a common pattern out of those explanations. Moreover, in some cases talk of acceptance is not only unnecessary, but even counter-intuitive: in the construction example, is it really helpful to say that the subject accepts that the costs will skyrocket, but does not believe so? It seems much more perspicuous to say that the subject does not want to take any risk that the costs will skyrocket, even if he believes unlikely that this will happen.

To sum up: pragmatic acceptance, as discussed by Bratman, does not qualify either as an epistemic primitive (because more economical explanations are viable) or as a molar notion (because it lacks a precise definition of its structure in terms of primitive components). Hence the suggestion that we should not include in epistemology a notion of acceptance conceived along these lines.

\section{AutomATICITY: WHY ROUTINES ARE NOT ACCEPTANCES}

Before moving to consider acceptance of conditional statements, another case of potential divergence between belief and action needs to be briefly discussed: behavioral routines. Imagine you are driving towards a new restaurant: at some point along the road, you realize that for some time you have been actually driving towards your home, 'as if' that was your destination. However, you did not believe anything of the sort - indeed, you 
always knew your destination was the restaurant. Similar occurrences, which are quite familiar to all of us, are not amenable of reconstruction in terms of beliefs and goals, as it was the case with Bratman's examples. However, should they count as acceptances? Would it make sense to say that, while driving, you are accepting your home to be the destination, even if you believe to be headed towards the restaurant?

The answer is "No", because there is a better explanation available: here cue-sensitive sub-personal processes take control of the behavior, with little or no role of conscious awareness at the personal level. In the driving example, the behavioral routine happens to jeopardize the agent's conscious plans, and this is why we consider the driver's behavior defective, i.e. somehow sub-par, in contrast with the cases discussed by Bratman. But the question is whether the notion of "normative rationality" should have legislation over such cases: the driver's action is not determined by some defective practical reasoning (e.g. a mistaken inference), but it is rather the result of a sub-personal process gone awry: a partial overlapping between the road to the restaurant and the road to home, or the time of day when the driving was taking place, triggers an automatic response that is inadequate to the present context. If this happens frequently enough, the adaptive value of the agent's automatic responses may be questioned: but this has little to do with the issue of normative rationality, that simply does not apply in the absence of explicit reasoning.

This is why framing similar cases in terms of acceptance vs. belief sounds so odd: these categories apply to practical reasoning, and serve to make sense of the subject's actions in terms of reasons. When reasons do not play any role to start with, as it happens with behavioral routines, talk of acceptance and belief is beside the point. It is worth noting that psychological research on automaticity (Bargh, Chartrand 1999; Gollwitzer 1999; Wegner 2002; Gollwitzer, Bargh 2005) suggests that behavior is frequently controlled at such implicit, sub-personal level. On this ground, it would be easy jumping to the conclusion that a reason-based explanation is rarely (if ever) appropriate for the analysis of human behavior. This conclusion is, however, largely unwarranted by current evidence on automaticity. What is warranted, instead, is careful consideration of the appropriate explanatory level and action granularity for reason-based explanations: in the driving example, the action of driving towards home is not reason-based and should not be assessed as such; but the realization that this is a mistake, and your consequent U-turn, are based on reasons, thus perfectly liable of analysis in terms of mental attitudes (see also Koriat 2007; Pacherie 2008). 
To sum up: acceptance is not a useful concept to explain cases where behavioral routines lead the agent to act in spite of some beliefs. Reference to automatic or semi-automatic processes of action control is much more adequate to explain both the observed behavior, and its divergence from some of the subject's beliefs. So there is nothing in behavioral routines that support inclusion of acceptance in descriptive epistemology.

\section{ACCEPTING CONDITIONALS AND CONDITIONAL DISPOSITIONS}

The traditional way of connecting acceptance of conditional statements to belief systems is in terms of the Ramsey test. According to Ramsey, "if two people are arguing 'If $p$ will $q$ ?' and are both in doubt as to $p$, they are adding $p$ hypothetically to their stock of knowledge and arguing on that basis about $q[\ldots]$. We can say they are fixing their degree of belief in $q$ given $p$ " (1929, p.155). Stalnaker offers the following, more precise formulation: "This is how to evaluate a conditional: First, add the antecedent (hypothetically) to your stock of beliefs; second, make whatever adjustments are required to maintain consistency (without modifying the hypothetical belief in the antecedent); finally, consider whether or not the consequent is then true" (1968, p.102).

Broadly speaking, two formalizations of the Ramsey test are possible, depending on whether belief states are modeled as closed sets of propositions, e.g. in AGM belief revision (Alchourrón et al. 1985; Gärdenfors 1988; Rott 2001), or as subjective degrees of probabilities, e.g. in Bayesianism (Howson, Urbach 1993). These approaches propose, respectively, a qualitative and a quantitative version of the Ramsey test, as follows (Leitgeb, in preparation):

Qualitative Ramsey test:

For every belief set $K$, for all sentences $A, B$ :

"If $A$ then $B$ " is acceptable (in $K$ ) if and only if $B \in K^{*} A$ where belief sets are deductive closed sets of sentences and the belief revision operator * obeys the AGM axioms (Gärdenfors 1988). 
Quantitative Ramsey test:

For every subjective probability measure $P$, for all sentences $A, B$ (with $P(A)>0$ ):

The acceptability of "If $A$ then $B$ " (in $P$ ) equals $P(B \mid A)$

where $P$ obeys the standard axioms of the probability calculus, and conditionals probabilities are defined by the ratio formula.

In short, the Ramsey test rules that a conditional "If $A$ then $B$ " is rationally acceptable (with degree $x$ ) in a belief state if and only if, were the belief state revised with $A$ as a new piece of evidence, then it would be rational to believe $B$ (with degree $x$ ). The relevant question for present discussion is how this acceptability should be interpreted: in particular, does acceptance of a conditional statement consists in believing the corresponding conditional? In pseudo-formal terms:

$$
\text { (1) } \operatorname{Acc}(A \rightarrow B)=\operatorname{Bel}(A \rightarrow B)
$$

If this was the case, then two other facts would follow, by applying (1) to both versions of the Ramsey test:

(2) $\quad(A \rightarrow B) \in K$ iff $B \in K^{*} A$

(3) $\quad P(A \rightarrow B)=P(B \mid A)$

It has been argued (Leitgeb 2007) that interpreting acceptance of a conditional as a belief in that conditional is not a viable option, due to impossibility results in AGM belief revision (Gärdenfors 1986; 1988) and in probability theory (Lewis 1976; 1986). These results prove that (2) and (3) are incompatible with, respectively, basic rationality assumptions in AGM belief revision and the standard axioms of probability theory, under minimal non-triviality assumptions. ${ }^{2}$ Hence accepting a conditional cannot be reduced to believing the corresponding conditional statement: in other words, (1) is false. This leaves open the possibility that acceptance of conditionals

\footnotetext{
${ }^{2}$ These results are very robust in probability theory, since they hold also for various "naturally" restricted classes of probability measures (Lewis 1976; 1986; Hájek, Hall 1994; Milne 1997; Bradley 2000). It remains to be seen whether triviality results in AGM belief revision are equally robust: it would be interesting to check whether Gärdenfors impossibility theorem holds also for belief revision operators which obey to only some of the standard AGM postulates, e.g. in non-prioritized belief revision (Hansson 1999).
} 
may constitute a genuinely independent epistemic attitude, worthy of inclusion in our epistemology.

Leitgeb (2007) proposes a different solution: acceptance of conditionals is taken to indicate conditional beliefs, as opposed to beliefs in conditionals, where the former are defined as higher-order single-track dispositions. The fact that subject $X$ accepts the conditional "If $A$ then $B$ " says something about the cognitive dispositions of $X$ : roughly speaking, it is equivalent to saying that, were $X$ to believe $A$ (with a certain degree), then he would also believe $B$ (with a certain degree). This analysis is restricted to indicative conditionals, whereas subjunctive conditionals (to which the Ramsey test does not apply) are taken to represent regularities in the world and thus be endowed with truth values (Leitgeb in preparation). Conditional beliefs are considered higher-order dispositions because they express the disposition of the subject to acquire mental states that are in turn amenable of dispositional analysis, e.g. a belief that $B$. Moreover, whereas simple beliefs are multi-track dispositions, i.e. they constraint the subject's behavior in various ways, conditional beliefs entail "a so-called singletrack disposition, i.e., a disposition to show one particular type of manifestation in one particular type of circumstance" (Leitgeb 2007, p.124; on the single-track/multi-track distinction, see Ryle 1949). Finally, conditional beliefs, as opposed to simple beliefs, have more than one propositional content and lack truth conditions (ibid., pp.122-123), and their communicative purpose is to express, as opposed to represent, the mental dispositions of the subject (Leitgeb in preparation; on the need for distinguishing metacognition from metarepresentation, see also Proust 2007).

Leitgeb is aware that some might resist his proposal of considering conditional beliefs as a species of the genus "belief": the obvious alternative would be to abandon any talk of belief in relation to the acceptance of conditionals, and claim that what we are dealing with in these cases is a different kind of attitude altogether (for suggestions in that vein, see Levi $1988 ; 1996$; Mellor 1993). Leitgeb regards this issue as largely terminological, and thus not worthy of too much attention (2007, p.120), albeit he endeavors to show that conditional beliefs present some typical features of belief (namely, intentionality, influence over action, representational structure, and justification), while lacking others, i.e. uniqueness of content and truth-aptness (ibid., pp.122-131). I agree with Leitgeb that the issue is largely terminological, in the sense that it does not matter much whether we label our attitudes on indicative conditionals as either "acceptances" or "conditional beliefs", provided we have good reasons to distinguish them 
from beliefs simpliciter. Nonetheless, in what follows I will refer to Leitgeb's conditional beliefs as acceptances, since I agree with Mellor and Levi that truth-aptness is paramount in determining our intuitions on belief, so that labeling "beliefs" some attitudes that admittedly lack that feature is, to my mind, rather counterintuitive. Moreover, acceptance is a vague notion in search of a precise definition, possibly one that does not reduce it to a mere shorthand. In the attitude expressed by assenting to indicative conditionals we have, at last, a suitable candidate.

The suggestion is thus to consider acceptances as conditional dispositions: accepting a certain state of affairs $X$ means being disposed to believe $X$ and/or act on $X$, but only when certain conditions occur. In cases like those considered by Leitgeb, both condition and disposition concern beliefs: accepting "If $A$ then $B$ " means being disposed to believe $B$, on condition that one believes $A$. But it is now possible to extend this definition to cover also cases of pragmatic acceptance a la Bratman: here the disposition is to use a certain propositional content in guiding one's action, on condition that certain circumstances obtain. What these circumstances are depends on the situation: in the construction example, the risk of facing bankruptcy is the key factor in making the subject disposed to act on the worst possible scenario; in the suspected friend example, loyalty and friendship are the condition that justify an inclination to grant the benefit of doubt in certain contexts (but not in others); in the war zone example, the soldier's belief that giving in to despair is useless and even despicable is the precondition for his disposition to bracket the worst possible scenario in his daily deliberation, regardless the fact that he sees that scenario as highly probable. In other words, what Bratman conceived as atomic instances of pragmatic acceptances are revealed to be the consequents of a series of conditional acceptances, where the antecedent is either left implicit or described as "context".

In fact, analyzing acceptances as conditional dispositions gives us a precise understanding of their characteristic context-sensitivity: the reason why we accept something (i.e. the consequent of a conditional acceptance) in certain contexts and not in others is because only those contexts satisfy the preconditions upon which our acceptance is based (i.e. the antecedent of a conditional acceptance). The mental attitude of acceptance per se is not context-dependent, but its application is: the subject, if rational, accepts "If a dear friend is in legal trouble and you are planning a social event, then do not ostracize him/her" in all contexts, but this (conditional) acceptance modifies the subject's behavior only when some dear friend is indeed in 
legal trouble and only as far as social events are concerned - it does not apply in a court of law, and yet this is no reason to claim that the subject no longer has the same conditional acceptance of what is appropriate to do when your friends are accused of a crime. ${ }^{3}$

So this view has the merit of reconciling the context-sensitivity of acceptances with a decent level of cognitive stability: rational agents do not change their mindset from one context to another, but part of their mindset is intrinsically conditional, thus it invites certain responses only in certain contexts. The implication is that all pragmatic acceptances worth considering can be understood as conditional dispositions where the antecedent is left either implicit or vague: this strategy works fine with Bratman's examples, as discussed. The difference with Leitgeb's view is that, in accepting conditional statements, the disposition expressed by the consequent concerns belief, whereas with pragmatic acceptance the disposition is directly tied with action. But of course a disposition towards action entails also a disposition to believe that such action will occur, assuming the antecedent of the conditional disposition is believed - and assuming the agent is minimally rational. ${ }^{4}$ In the accused friend example, believing that my friend is accused and that I am planning a social event has two consequences: I will not ostracize him/her, and I believe I will not ostracize him/her. The second consequence is precisely what is required by Leitgeb's analysis of conditional beliefs: hence extending a similar analysis to pragmatic acceptances is not in contrast with Leitgeb's proposal, ${ }^{5}$ but it provides yet an-

\footnotetext{
${ }^{3}$ Notice that acceptance here refers to mental attitudes, not linguistic behavior. Take a subject who is, as a matter of fact, disposed to act on $B$ when $A$ is believed: it is immaterial whether or not the subject is ready to linguistically assent to the corresponding conditional "If $A$, then act accordingly to $B$ ". Indeed, the subject could well be unaware of having such a conditional disposition, thus being incapable of assenting to the corresponding conditional: nonetheless, as long as the disposition is present, it correctly describes the mental attitude of the agent, and thus we are justified in saying that the subjects accepts " $B$, if $A$ ".

${ }^{4}$ Ceteris paribus conditions may also be needed to ensure that the implication is carried through. For the sake of brevity, I do not discuss the issue here: see Leitgeb (2007, pp.121-125) for some considerations on ceteris paribus conditions in the context of accepting indicative conditionals.

${ }^{5}$ This means that Leitgeb's argument against interpreting acceptance of a conditional as a belief in that conditional applies also to pragmatic acceptances, once conceived as conditional dispositions. Given a pragmatic acceptance of the generic form "If $A$, then act according to $B$ ", this is an indicative conditional, to which Gärdenfors' and Lewis' impossibility results still apply: it cannot be the case that having such a conditional
} 
other reason to use the label "acceptance" rather than "belief" in this context.

Stalnaker's intuitive definition of acceptance resonates with the current proposal: "To accept a proposition is to treat it as a true proposition in one way or another - to ignore, for the moment at least, the possibility that it is false. [...] To accept a proposition is to act, in certain respects, as if one believed it" (Stalnaker 1984, pp.79-80). Some vague expressions in this characterization ("in one way or another", "in certain respects") hint to the conditional nature of acceptance: only when certain conditions apply the corresponding disposition (to act and/or to believe) is elicited. But to insist that only the latter constitutes an acceptance, without appreciating its conditional nature, would undermine the heuristic value of this notion, and leave it open to the criticisms discussed in section 3. Moreover, conceiving acceptances as conditional dispositions gives them a clear place in a dispositional epistemology, where beliefs are rather conceived as dispositions simpliciter, i.e. unconditional dispositions. ${ }^{6}$ Believing $p$ means being ready to act according to $p$ no matter what. In contrast, accepting $p$ (i) is just a shorthand for "accepting ( $p$ if $q$ )" and (ii) means being ready to act according to $p$ only when $q$ is believed - where $q$ stands for any proposition of arbitrary complexity that serves to capture the circumstances upon which being disposed to $p$ is conditional. ${ }^{7}$

disposition is tantamount to believing the corresponding conditional, unless we want to forsake minimal rationality.

${ }^{6}$ It would be tempting here to postulate a straightforward connection with Ryle's distinction between multi-track and single-track dispositions: the claim would be that what makes a disposition multi-track, i.e. manifest across different contexts and in a variety of ways, is its being unconditional, whereas conditional dispositions are necessarily single-track, i.e. tied to specific conditions for their manifestation. This, however, would misrepresent Ryle's distinction, which is about the ways in which a disposition manifests itself (see for instance 1949, pp.43-44), and not so much about the conditions that trigger its manifestation. In short, single-track/multi-track is about how a disposition is manifested, whereas unconditional/conditional is about when it manifests. If there is a relationship between these couples of notions, it is not in terms of identity or implication.

${ }^{7}$ A formal consequence is that believing $p$ entails having an infinite number of acceptances of the form "If $X$, then act on $p$ ", where $X$ is an arbitrary statement. Since believing $p$ means being disposed to act on $p$ no matter what, it is true that I am disposed to act on $p$ given whatever circumstances $X$ might obtain, hence I can be said to be in a conditional state of acceptance of the form "If $X$, then act on $p$ ". In short: an unconditional disposition to $p$ is equivalent to having an infinite number of conditional disposition to $p$ with whatever you like as antecedent. Should we be alarmed by such profli- 
The strong claim that characterizes this view is that there is no such thing as "unconditional acceptance" of a given proposition: all acceptances are conditional by definition, whether the relevant conditions are spelled out or left implicit. This is not mere stipulation, but rather an appeal to intuition: give me a single example of acceptance that is neither conditional to specific circumstances nor reducible to belief, and the definition of acceptance as conditional disposition will be falsified. But no such counterexample is in view: none of Bratman's cases qualifies, and I honestly cannot think of any other that would. If a proposition is endorsed with no strings attached, then it is believed, and there is no reason to invoke the weaker notion of acceptance for it - as discussed in section 2, to reject the view of factual statements as "theories-in-a-nutshell". If, on the other hand, my endorsement is conditional upon certain circumstances, then the proposition cannot be said to be believed, because belief has no such qualms: as Bratman would say, it is not context-dependent in that way. ${ }^{8}$ In this case, and only in this case, it is useful to apply the notion of acceptance. Finally, when the proposition is never endorsed, regardless the circumstances, then it is simply disbelieved, that is, neither believed nor accepted.

Given this view of acceptance, let us see how it fares as a candidate for inclusion in epistemology. The first question is whether this kind of acceptance is a primitive or a molar notion. At first sight, it may seem a molar concept, since belief figures as part of its definition: accepting " $p$, if $q$ " means being disposed to $p$ (and to believe you $p$ ) if you believe $q$. But the role of belief in this definition is not that of a constitutive element: acceptance, thus conceived, is not reducible to belief, or to belief plus action. Belief is not a necessary condition for acceptance: it remains true that I accept " $p$, if $q$ " (i.e. I am disposed to $p$ on condition that $q$ ) even if $q$ never

gacy of acceptances? Not at all, because acceptances describe what a given cognitive system is geared to do under certain circumstances, but they do not represent mental content. If we decide to be needlessly byzantine in how we describe the workings of the system, this is a problem for our description, not for the system itself. The fact that $X$ believes $p$, i.e. $X$ actions are guided by $p$ in all contexts, has the same degree of complexity whether you choose to describe it as $X$ having a single unconditional disposition to $p$ or as $X$ having an infinite number of conditional dispositions to $p$. Since profligacy of acceptances is a threat only for theoretical reconstruction, it can be handled at that level, e.g. forbidding translation of unconditional dispositions as infinite sets of conditional dispositions.

${ }^{8}$ An obvious corollary of this view is that Leitgeb's "conditional beliefs" are actually a specific class of (non-pragmatic) acceptances. The reasons for this terminological disagreement have already been discussed. 
comes to be believed and so my disposition is never realized. Instead, believing $q$ and thus doing $p$ is a sufficient condition for acceptance, in the sense that it provides an instantiation of it: but acceptance qua conditional disposition does not require such instantiation for being correctly attributed to the subject. So I propose to conceive acceptance as a primitive notion, albeit quite different in nature from belief: whereas acceptances are conditional dispositions that express the cognitive potentialities of the subject, beliefs are unconditional dispositions that mirror the subject's understanding of reality. ${ }^{9}$ The former tell us something of how the subject's mind works, the latter refer to how the world is supposed to be, according to the subject.

But what kind of primitive notion is acceptance - epistemic, conative, or a mix of both? Part of the answer depends on the kind of acceptance being considered. Pragmatic acceptances, like those dear to Bratman, have a clear conative element, insofar as their consequent specifies an action policy: being cautious in allocating money for your construction project, being supportive of your friends, etc. Acceptance of indicative conditionals in general, however, determines what the subject would believe, if the antecedent of the conditional was also believed: as such, it seems to qualify as an epistemic attitude and not a conative one, since believing is traditionally conceived as an event rather than an action. However, the whole distinction epistemic/conative may be largely misleading here, since it refers most naturally to mental states, whereas acceptances refer instead to mental transitions - or, more precisely, predispositions to realize a given transition in the appropriate circumstances. Can a transition be described as either "epistemic" or "conative", and what purpose would this serve? At best, we can say that the conditions triggering such transitions are epistemic (beliefs), while the end result can be either conative (an action) or epistemic (a belief), or both. None of this, however, seems sufficient to characterize the transition itself and the corresponding conditional disposition as either epistemic or conative.

In conclusion, it seems better to abandon the distinction between epistemic and conative attitude, and characterize acceptance rather as a primitive transformational attitude, i.e. a notion that describes how a cognitive system is expected to react, in the presence of specific inputs. If so, should we grant it admittance in our descriptive epistemology, on the grounds of van Fraassen's initial invitation? This time the answer is positive, but with

\footnotetext{
${ }^{9}$ This distinction is different from, but largely compatible with, Audi's notion of dispositions to believe (1994).
} 
a proviso: as soon as acceptance qua conditional disposition is given credit in our epistemology, on a par with belief, this forces us to expand the boundaries of that epistemology beyond merely epistemic attitudes, at least as they are traditionally conceived. Acceptance is not just a slightly different way of endorsing a given proposition: it is rather a different matter altogether, a principle of organization (and functioning) of the cognitive system to which it refers. Among other things, it invites us to embrace a dispositional epistemology, for both beliefs (as unconditional dispositions) and acceptances (as conditional dispositions). This has important consequences for the scope and purposes of epistemology, which will need to be carefully considered in future works on this topic.

\section{CONCLUSIONS}

After critical scrutiny, two well-known versions of acceptance, van Fraassen's acceptance of scientific theories and Bratman's pragmatic acceptance, had to be denied the status of primitive epistemic notions. What they can be, for partially different reasons, is a shorthand for naming complex structures of beliefs and goals. The usefulness of such shorthand, however, is questionable. Along the way of this critique, we noted also that cases where actions and beliefs differ due to automatic sub-personal routines do not count as instances of acceptance either. On a more positive note, acceptance of conditional statements gave us more epistemological leverage, helping to reconsider also pragmatic acceptance in a new light. This led us to define acceptances as conditional dispositions, and to study their properties and their place in a dispositional epistemology.

This may have far-reaching consequences for epistemology in general: in particular, a conditional view of acceptances naturally invites adopting a dispositional epistemology also for beliefs. The morale is that the step from open-minded descriptive epistemology to radical re-description of traditional epistemology may be short. Is this move justified by the arguments produced so far? Should the pebble of acceptance start an epistemological avalanche? Answering this question requires considerations that go beyond the aim of this essay. But a conditional conclusion is offered for that debate. If we want to have a meaningful notion of acceptance, then embracing a dispositional view of epistemology seems to be the only way to go. If, on the other hand, we are not yet ready to bring our dispositions out of the closet, then we are left with an impoverished notion of 
acceptance, one that at best qualifies as a shorthand of dubious value. According to the line of reasoning presented in this paper, these are the options. Whatever we pick, we must know (and accept) the consequences.

\section{ACKNOWLEDGMENTS}

This work was supported by the CNR and the EC $6^{\text {th }}$ Framework Programme as part of the ESF EUROCORES Programme CNCC. I am grateful to Hannes Leitgeb, Mehmet Cakmak, and Cristiano Castelfranchi for extensive discussion of the ideas presented here, and to participants of the CNCC workshop on "Representations: Perspectives from philosophy, psychology and neuroscience" at the $31^{\text {st }}$ International Wittgenstein Symposium (Kirchberg am Wechsel, 11 August 2008).

\section{REFERENCES}

Alchourrón, C., Gärdenfors, P., Makinson, D. (1985). "On the Logic of Theory Change: Partial Meet Contraction and Revision Functions". Journal of Symbolic Logic 50, pp.510-530.

Audi, R. (1994). "Dispositional Beliefs and Dispositions to Believe". Noûs 28 (4), pp.419-434.

Bargh, J., Chartrand, T. (1999). "The Unbearable Automaticity of Being". American Psychologist 54, pp.462-479.

Bradley, R. (2000). "A Preservation Condition for Conditionals". Analysis 60 (3), pp.219-222.

Bratman, M. (1992). "Practical Reasoning and Acceptance in a Context". Mind 101, pp.1-15.

Cohen, L. (1989). "Belief and Acceptance". Mind 98, pp.367-389.

Engel, P. (1998). "Believing, Holding True, and Accepting". Philosophical Explorations 1, pp.140-151.

Gärdenfors, P. (1986). "Belief Revisions and the Ramsey Test for Conditionals". Philosophical Review 95, pp.81-93.

Gärdenfors, P. (1988). Knowledge in Flux: Modelling the Dynamics of Epistemic States. Cambridge: MIT Press.

Gilbert, M. (2002). "Belief and Acceptance as Features of Groups". Protosocio$\log y 16, \mathrm{pp} .35-69$.

Gollwitzer, P. (1999). "Implementation Intentions: Strong Effects of Simple Plans". American Psychologist 54, pp.493-503.

Gollwitzer, P., Bargh, J. (2005). "Automaticity in Goal Pursuit". In: A. Elliot, C. Dweck (eds.), Handbook of competence and motivation. New York: Guilford Press, pp.624-646. 
Hájek, A., Hall, N. (1994). "The Hypothesis of the Conditional Construal of Conditional Probability". In: E. Eells, B. Skyrms (eds.), Probability and Conditionals. Belief Revision and Rational Decision. Cambridge: Cambridge University Press, pp.75-111.

Hansson, S. (1999). "A Survey on Non-prioritized Belief Revision". Erkenntnis 50, pp. $413-427$.

Koriat, A. (2007). "Metacognition and Consciousness"s. In: P. Zelazo, M. Moscovitch, E. Thompson (eds.), The Cambridge Handbook of Consciousness. Cambridge: Cambridge University Press, pp.289-325.

Leitgeb, H. (2007). "Beliefs in Conditionals vs. Conditional Beliefs". Topoi 26, pp.115-132.

Leitgeb, H. (in preparation). "Metacognition and Indicative Conditionals". Draft manuscript.

Levi, I. (1988). "Iteration of Conditionals and the Ramsey Test". Synthese 76, pp.4981.

Levi, I. (1996). For the Sake of the Argument. Ramsey Test Conditionals, Inductive Inference, and Nonmonotonic Reasoning. Cambridge: Cambridge University Press.

Lewis, D. (1976). "Probabilities of Conditionals and Conditional Probabilities". Philosophical Review 85, pp.297-315.

Lewis, D. (1986). "Probabilities of Conditionals and Conditional Probabilities II". The Philosophical Review 95, pp.581-589.

Mellor, D. (1993). "How to Believe a Conditional". The Journal of Philosophy 90 (5), pp.233-248.

Milne, P. (1997). "Quick Triviality Proofs for Probabilities of Conditionals". Analysis 57 (1), pp.75-80.

Pacherie, E. (2008). "The Phenomenology of Action: A Conceptual Framework". Cognition 107 (1), pp.179-217.

Proust, J. (2007). "Metacognition and Metarepresentation: Is a Self-directed Theory of Mind a Precondition for Metacognition?". Synthese 159, pp.271-295.

Ramsey, F. (1929). “General Propositions and Causality”. In: D. Mellor (ed.), F. Ramsey, Philosophical papers. Cambridge: Cambridge University Press, 1990, pp.145-163.

Rott, H. (2001). Change, Choice and Inference: A Study of Belief Revision and Nonmonotonic Reasoning. Oxford: Oxford University Press.

Ryle, G. (1949). The Concept of Mind. London: Hutchinson.

Stalnaker, R. (1968). "A Theory of Conditionals". In: N. Rescher (ed.), Studies in Logical Theory. Oxford: Blackwell.

Stalnaker, R. (1984). Inquiry. Cambridge: MIT Press.

Tuomela, R. (2000). "Belief versus Acceptance". Philosophical Explorations 2, pp.122-137.

van Fraassen, B. (1980). The Scientific Image. New York: Oxford University Press.

van Fraassen, B. (2001). "Constructive Empiricism now". Philosophical Studies 106, pp.151-170.

Wegner, D. (2002). The Illusion of Conscious Will. Cambridge: MIT Press.

Wray, K. (2001). "Collective Belief and Acceptance”. Synthese 129, pp.319-333. 
